\title{
A Rapid-Response Outpatient Model for Reducing Hospitalization Rates Among Suicidal Adolescents
}

Brian Greenfield, M.D.

Charles Larson, M.D.

Lily Hechtman, M.D.

Cecile Rousseau, M.D.

Robert Platt, Ph.D.

Objective: The authors studied the clinical outcomes of suicidal adolescents who were treated within a rapid-response outpatient model in a setting in which a ten-day wait was usually required before outpatient treatment could be started, leaving hospitalization as the only immediately available alternative. Methods: A total of 286 suicidal adolescents aged 12 to 17 years who came to the emergency department of a pediatric hospital were assigned to receive rapid-response outpatient follow-up (the experimental group) or to a control group. Demographic and clinical data were obtained at baseline, and outcomes data were obtained at two and six months. Results: The demographic and clinical characteristics of the two groups were similar at baseline. Hospitalization rates in the experimental and control groups, respectively, were 10 percent and 40 percent at baseline, 17 percent and 41 percent at twomonth follow-up, and 18 percent and 43 percent at six-month follow-up, corresponding to a relative risk of hospitalization of .41 in the experimental group at six months. No between-group differences were observed in changes in levels of suicidality or in overall functioning over the follow-up period, and none of the patients had died at six months. Conclusions: Suicidal adolescents who received rapid-response outpatient follow-up had a lower hospitalization rate than those who did not. The two groups achieved similar increases in levels of functioning and decrease in levels of suicidality, suggesting that suicidal adolescents can be treated within a rapid-response outpatient model and thus avoid hospitalization. (Psychiatric Services 53:1574-1579, 2002)

\footnotetext{
A dolescent suicidality is disturbingly common, with annual estimates of suicide attempts among 15-to 24-year-olds sur-
}

passing one million in the United States and 130,000 in Canada (1). These estimates correspond to a rate of completed suicides in 1997 of 11.4

Dr. Greenfield is principal investigator and assistant professor of psychiatry and of pediatrics, Dr. Larson is associate professor of pediatrics and of epidemiology, biostatistics, and occupational health, Dr. Hechtman is professor of psychiatry and of pediatrics, and Dr. Rousseau is associate professor of psychiatry at McGill University School of Medicine in Montreal. Dr. Platt is assistant professor of pediatrics and of epidemiology and biostatistics at McGill University Health Center. Send correspondence to Dr. Greenfield at the Montreal Children's Hospital, 2300 Tupper Avenue, Montreal, Quebec, Canada H3H 1P3 (e-mail, brian.greenfield@muhc.mcgill.ca). The results of this study were presented as a poster at the annual meeting of the American Academy of Child and Adolescent Psychiatry held October 19-24, 1999, in Chicago. per 100,000 in the United States (2), 13.7 in Canada, and 22.1 in the province of Quebec (3), with females being at greatest risk (4). Some suicidal adolescents require the security of hospitalization (5), although the stresses associated with hospitalization can disrupt the overall lifestyles of the adolescents and their parents (5).

We report on the outcomes of suicidal adolescents who came to the emergency department of a university hospital and were treated with one of two treatment models. The first model was a rapid-response outpatient team approach associated with a decreased hospitalization rate. The second model involved a wait of approximately ten days before follow-up could be initiated on discharge from the emergency department, often requiring psychiatrists to hospitalize the patient.

In light of trends to decrease hospitalization rates among youths (6), it is important to determine what constitutes appropriate management of this patient population. Many factors influence the decision to hospitalize a suicidal adolescent (7), such as individual characteristics of the adolescent (8-13), the availability of community resources, and concerns about compliance with treatment plans (14). These factors have led to wide variations in admission rates (44 percent to 81 percent) (15), depending on regional standards.

Studies of outpatient-based crisis intervention models for these patients have had methodologic limita- 
tions, such as the absence of a condition-either control or experimental-that is clearly identified as constituting hospitalization (5,16-19), small samples $(5,20)$, the absence of standardized measures (5), under- or overrepresentation of patients with comorbid conditions $(16,17,19)$, recruitment of only females (18), high dropout rates (19), the use of a hypothetical treatment condition (20) or of a protocol requiring highly specialized training to administer the intervention (5), and the inclusion of both adolescents and young adults $(17,19)$.

In one report, home-based multisystemic family therapy was suggested as a safe alternative to hospitalization (21), but the study was limited by an overrepresentation of patients of lower socioeconomic status, hospitalization of a large number of patients in the experimental group (44 percent), failure to note suicidality levels, and the use of an intervention that had limited generalizability because it was highly specialized.

In this prospective study we addressed some of these limitations by including suicidal adolescents of both sexes, without exclusionary psychiatric diagnoses, and from every socioeconomic class and by using a consistently applied methodology. Outcome measures were administered in person (including a measure of suicidality), and patients were assigned either to a rapid-response outpatient model (the experimental group) or to a control condition in which there was greater reliance on hospitalization. We hypothesized that the suicidal adolescents in the experimental group would have similar clinical outcomes to those in the control group but with a lower rate of hospitalization.

\section{Methods \\ Sample}

The study was conducted in one of two pediatric emergency departments that serves a population of 3.5 million people and that has an average of more than 1,500 visits weekly. Approximately .5 percent of these patients are referred for a psychiatric assessment; of these, approximately 50 percent are suicidal adolescents. Seventy-three percent of the patients in this study spoke English at home, 23 percent spoke French, and 4 percent spoke another language. This study examined the full range of suicidal adolescents, from those with suicidal ideation alone to those who had made serious suicide attempts.

\section{Procedure}

During the study period (December 1996 to October 1998), 344 suicidal adolescents aged 12 to 17 years came to the emergency department and were assessed by the on-call pediatrician as having experienced a suicidal event that required an immediate psychiatric consultation. Forty-one of them (12 percent) were hospitalized for medical or surgical reasons, as determined by the pediatrician, and thus were excluded from the study, leaving 303 (88 percent). Seventeen of these adolescents (5 percent) refused to participate in the study, for a total of 286 participants. A research assistant informed each patient and his or her parent of the study's structure and aim and sought their informed consent to participate, after which they were followed up for six months. Approval for the study was obtained from the institutional review board of the Montreal Children's Hospital.

The patients were assigned to the experimental group or the control group, depending on the group assignment of the psychiatrist who treated them when they came to the emergency department. For each group, there was a group of psychiatrists on call that worked only with patients assigned to that group. A prearranged on-call schedule ensured that both treatment conditions were balanced in terms of total duration, times of the day (morning, afternoon, evening, or night), days of the week (including weekends), weeks of the month, and months of the year during both study years. This approach was used to ensure that there would be essentially no difference between the two groups. For the experimental group, the on-call psychiatrists had access to the rapid-response outpatient team, whereas the psychiatrists working with patients in the control group did not. Psychiatrists in both groups assessed the referred adolescents and their family members in the emergency department for psychi- atric diagnosis and suicidal risk and could hospitalize the patients.

Each patient was given $\$ 25$ to defray parking and other transportation expenses. Those who declined to participate in the study (17 patients) were assured that they would receive the same quality of care as the other patients. Immediately after the psychiatric assessment in the emergency department, which was attended by the research assistant, the research assistant administered a battery of semistructured interviews with the adolescent alone and then separately with one parent. The psychiatrist then either admitted the patient or discharged the patient from the emergency department with subsequent referral to the experimental condition or to the control condition. In addition to receiving clinical care, all patients were contacted for a research interview two and six months after the emergency department assessment.

The research assistants were not informed of the study hypothesis or of the psychiatrists' treatment group assignments. The psychiatrists each had at least four years of residency training, two years of which were in child psychiatry, and an average of ten years of posttraining clinical experience and represented a wide array of clinical orientations.

\section{Interventions}

The rapid-response outpatient team. The rapid-response outpatient team consisted of one part-time psychiatrist and one psychiatric nurse and was oriented toward outpatient care immediately after assessment in the emergency department. These team members initiated telephone contact with every referred patient and his or her family to plan a followup appointment. Only three patients (2 percent) missed their scheduled follow-up appointment.

Upon referral to this team by the experimental group psychiatrists, the patient and his or her family members were assessed to identify the nature of the crisis, the precipitating events, and the strengths and weaknesses of the adolescent's support system. Interventions were aimed at reframing any misconceptions, maladaptive behaviors, and communica- 


\section{Table 1}

Baseline demographic and substance use characteristics of suicidal adolescents in the rapid-response outpatient follow-up (experimental) group and the control group $^{\mathrm{a}}$

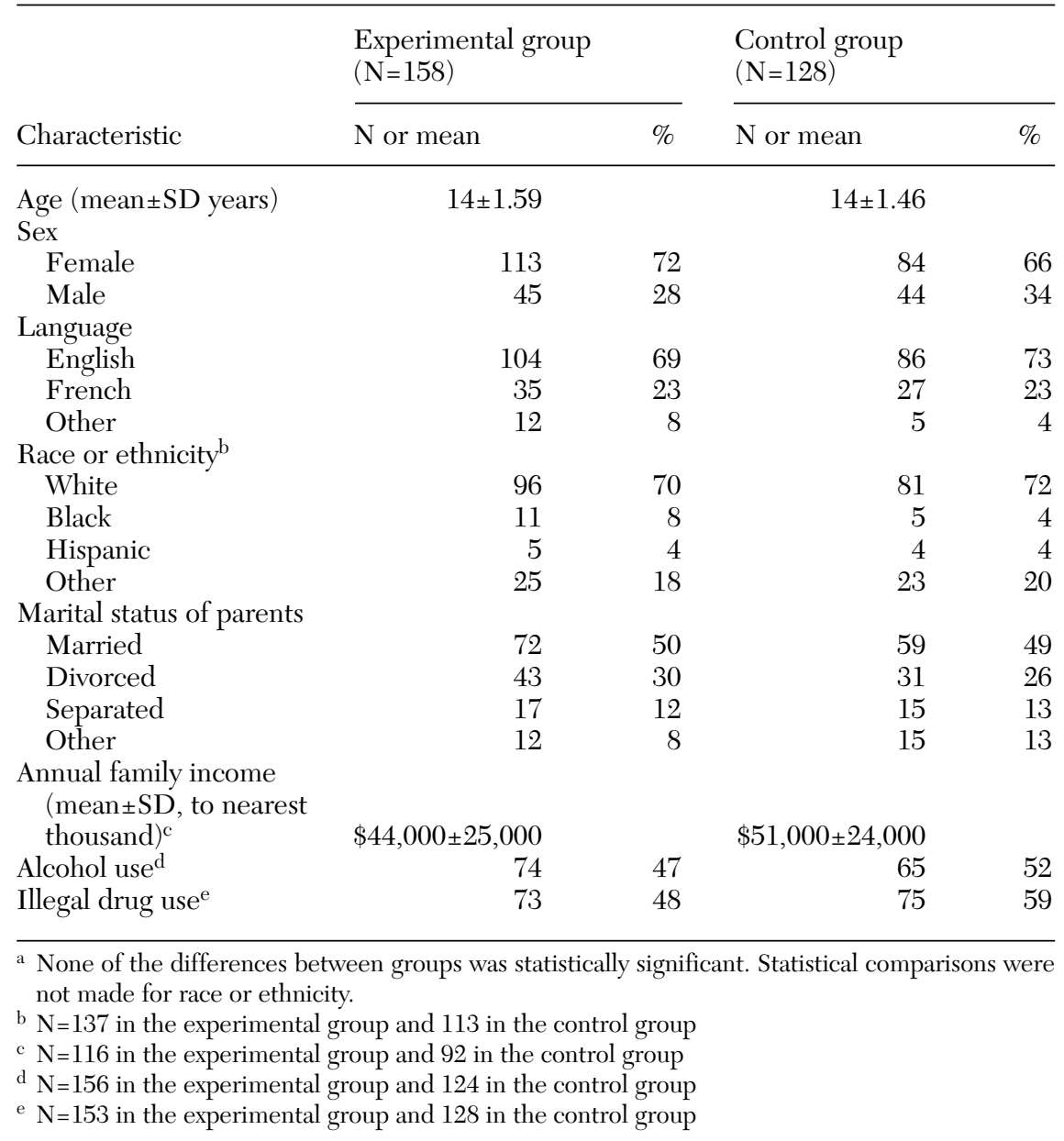

tion patterns that contributed to the patient's or the family's stresses. Medication was used when appropriate, and community resources were used when available.

The mean \pm SD and median number of contacts with the rapid-response team at two months were $4.18 \pm 4.49$ and 3.50 , respectively, and the mean duration of treatment was $6.35 \pm 4.11$ weeks. At six months, the mean and median number of contacts was $9.98 \pm 8.47$ and 9.00 , respectively, and the mean duration of treatment was $17.69 \pm 12.50$ weeks. Contact with the team continued until long-term follow-up was arranged in the community and was also available in case of future crisis.

Control group. To continue the treatment initiated in the emergency department, psychiatrists of patients in the control group could either hos- pitalize the patient, follow the patient as an outpatient, or refer the patient to a variety of community resources, such as a hospital-based outpatient psychiatric clinic, a non-hospital based community health facility, or a private mental health worker. A decision to hospitalize a patient in the control group was made by the control group psychiatrist who was oncall at the time the patient came to the emergency department.

Initial and subsequent hospitalizations. Many factors contributed to the psychiatrists' decisions to hospitalize patients, such as previous suicide attempts, previous hospitalizations, the lethality of the current attempt, persisting anger or intent to die, and the strength of support systems. It was anticipated that the experimental group's access to a rapidresponse outpatient team would de- crease that group's hospitalization rate (the descriptive variable). $\mathrm{Pa}$ tients retained their original group assignment if they returned to the emergency department.

\section{Measures}

All measures were administered to all patients and demonstrated acceptable reliability and validity, except for a substance abuse questionnaire created for this study (discussed below). The interview protocol at recruitment included the following seven measures and questionnaires. The Diagnostic Interview Schedule for Children (DISC) (22) was administered to the adolescent, specifically those sections dealing with DSM-III-R diagnoses (23) that commonly occur among suicidal adolescents-conduct and major affective disorders. The Children's Global Assessment Scale (CGAS) (24), adapted from the Global Assessment Scale for Adults, measured the patient's level of functioning. It is the third part of the Schedule for Affective Disorders and Schizophrenia for School-Age Children (KiddieSADS) (25), presents as a ten-category description of adaptive behaviors (level of functioning) along a 100point scale, and is guided in its scoring by questions about the patient's global functioning.

The Spectrum of Suicidal Behavior Scale (26), administered during a semistructured interview, measured suicidal behavior in a hierarchy along a 5 -point ordinal scale that includes no suicidal behavior, scored as 1; suicidal ideation, scored as 2; suicidal threats, scored as 3; mild suicide attempts, scored as 4; and serious suicide attempts, scored as 5. The Index of Family Relations (IFR) (27), administered to the patient, quantified the extent, severity, or magnitude of family problems. The Coddington Life Events Scale, a 40-item scale (28), measured the patient's and family's stressful and precipitating life events.

Sociodemographic information obtained ranged from the parents' education, income, job description, and marital status to the number of siblings. Finally, a substance use questionnaire was administered, which consisted of both a list of substances commonly used by adolescents and a 
scale to record the frequency of use of each substance.

The two-month follow-up evaluation (278 patients, or 97 percent of the original sample) included the CGAS; the Spectrum of Suicidal Behavior Scale; a separate, semistructured, 20-minute interview with the adolescent and a parent (usually the mother) about the patient's functioning (the CGAS); and notation of any interim hospitalizations.

Evaluation at the six-month followup (262 patients, or 92 percent of the original sample) included the DISC, the CGAS, the Spectrum of Suicidal Behavior Scale, and readministration of the 20-minute, two-month followup, clinical interview.

\section{Analysis}

The data collected were entered into a database and analyzed with SPSS$\mathrm{PC}$ and SAS software. Differences in means were assessed by using $t$ tests. Differences in proportions were assessed with chi square or Fisher's exact tests, depending on cell sizes. Treatment groups were compared by using relative risks and their associated 95 percent confidence intervals.

\section{Results}

A total of 158 patients were assigned to the experimental group, 18 (11 percent) of whom were immediately hospitalized and 131 (83 percent) of whom were referred to the rapid-response outpatient team for ongoing immediate care on discharge from the emergency department. Psychiatrists treated nine patients (6 percent) themselves without delay instead of referring them to the rapid-response team.

A total of 128 patients were assigned to the control group, 53 (41 percent) of whom were immediately hospitalized. The median number of days to the first telephone contact with a health care professional on discharge from the emergency department was 1.5 (lower quartile to upper quartile, 1.0 to 6.0 ) for the patients in the experimental group and four (1.0 to 7.5) for patients in the control group. The median time to the first appointment was six days (1.0 to 11.0) for the patients in the experimental group and ten days (5.0 to 17.0) for
Table 2

Baseline clinical characteristics of suicidal adolescents in the rapid-response outpatient follow-up (experimental) group and the control group ${ }^{a}$

\begin{tabular}{|c|c|c|c|c|}
\hline \multirow[b]{2}{*}{ Characteristic } & \multicolumn{2}{|c|}{$\begin{array}{l}\text { Experimental } \\
\text { group } \\
(\mathrm{N}=158)\end{array}$} & \multicolumn{2}{|l|}{$\begin{array}{l}\text { Control } \\
\text { group } \\
(\mathrm{N}=128)\end{array}$} \\
\hline & $\mathrm{N}$ or mean & $\%$ & $\mathrm{~N}$ or mean & $\%$ \\
\hline \multicolumn{5}{|l|}{ Suicidal thoughts } \\
\hline More than six months ago & 92 & 61 & 68 & 55 \\
\hline In the past six months & 110 & 72 & 98 & 80 \\
\hline \multicolumn{5}{|l|}{ Suicide attempts } \\
\hline More than six months ago & 51 & 33 & 35 & 29 \\
\hline In the past six months & 48 & 32 & 51 & 42 \\
\hline \multicolumn{5}{|l|}{ Emergency department assessment } \\
\hline CGAS score $(\text { mean } \pm S D)^{b}$ & $39 \pm 10.6$ & & $40 \pm 12.1$ & \\
\hline IFR score $(\text { mean } \pm \mathrm{SD})^{\mathrm{c}}$ & $25 \pm 18.0$ & & $22 \pm 16.2$ & \\
\hline Life events $(\text { mean } \pm \text { SD })^{\mathrm{d}}$ & $9 \pm 9.2$ & & $8 \pm 6.5$ & \\
\hline Depression $^{\mathrm{e}}$ & 73 & 46 & 64 & 50 \\
\hline Conduct disorder $^{\mathrm{f}}$ & 31 & 20 & 36 & 28 \\
\hline Suicidality score $(\text { mean } \pm \text { SD })^{g}$ & $2.5 \pm 1.2$ & & $2.7 \pm 1.2$ & \\
\hline
\end{tabular}

a None of the differences between groups was statistically significant.

b Children's Global Assessment Scale. Possible scores range from 1 to 100 , with higher scores indicating better functioning.

${ }^{c}$ Index of Family Relations. Possible scores range from 0 to 100, with higher scores indicating greater family dysfunction.

d Assessed with the Coddington life events scale. Possible scores range from 1 to 40, with higher scores indicating the occurrence of more stressful life events.

e Depression was assessed with the Diagnostic Interview Schedule for Children (section on major affective disorder).

${ }^{\mathrm{f}}$ Conduct disorder was assessed with the Diagnostic Interview Schedule for Children (section on conduct disorder).

g Suicide was assessed with the Spectrum of Suicidal Behavior Scale. Possible scores range from 1 to 5 , with higher scores suggesting more severe suicidal behavior. patients in the control group.

Tables 1 and 2 summarize the sociodemographic and clinical characteristics of the two treatment groups at recruitment. No between-group differences were found in family income, the primary language of the patients, or baseline diagnoses (including level of suicidality and functioning). The only difference between the two groups was in the use of drug overdose as a method of suicide attempt (10 percent in the experimental group and 23 percent in the control group,

\section{Table 3}

Suicide ideation and type of suicide attempt among suicidal adolescents in the rapid-response outpatient follow-up (experimental) group and the control group

\begin{tabular}{|c|c|c|c|c|}
\hline \multirow[b]{2}{*}{ Type of attempt } & \multicolumn{2}{|c|}{$\begin{array}{l}\text { Experimental } \\
\text { group }(\mathrm{N}=158)\end{array}$} & \multicolumn{2}{|c|}{$\begin{array}{l}\text { Control group } \\
(\mathrm{N}=128)\end{array}$} \\
\hline & $\mathrm{N}$ & $\%$ & $\mathrm{~N}$ & $\%$ \\
\hline Drug overdose* & 16 & 10 & 29 & 23 \\
\hline Ingestion of another substance & 2 & 1 & 1 & 1 \\
\hline Hanging & 1 & 1 & 5 & 4 \\
\hline Jumping from height & 6 & 4 & 0 & - \\
\hline Cutting or slashing & 24 & 15 & 15 & 12 \\
\hline Firearm & 0 & - & 1 & 1 \\
\hline Other & 8 & 5 & 7 & 6 \\
\hline Ideation only & 101 & 64 & 70 & 55 \\
\hline
\end{tabular}

* $\mathrm{p}<.004$ 


\section{Table 4}

Outcomes among suicidal adolescents in the rapid-response outpatient follow-up (experimental) group and the control group

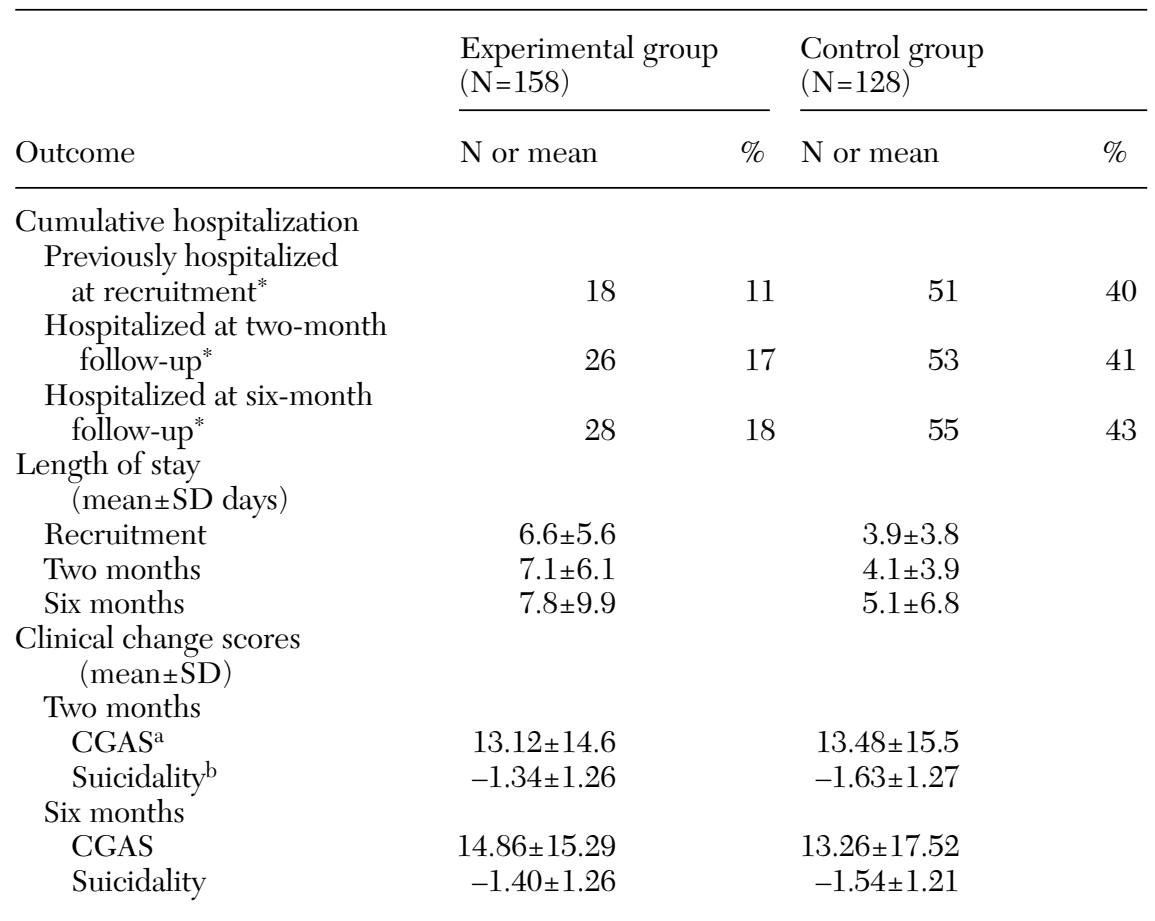

${ }^{a}$ Children's Global Assessment Scale; higher scores indicate better functioning.

b Spectrum of Suicidal Behavior Scale; higher scores suggest more severe suicidal behavior. Negative values indicate a change in the group mean from higher suicidality to lower suicidality, reflective of an improved clinical status.

* $\mathrm{p}<.001$

$\left.\chi^{2}=8.373, \mathrm{df}=1, \mathrm{p}=.004\right)($ Table 3$)$.

The hospitalization rate immediately after assessment in the emergency department was 11 percent in the experimental group and 40 percent in the control group (relative risk $[\mathrm{RR}]=.29,95$ percent confidence interval $[\mathrm{CI}]=.18$ to $.46, \mathrm{p}<.001$ ) (Table 4). The six-month overall hospitalization rate, defined as hospitalization at least once at any hospital for reasons related to suicidality, was significantly different between the two groups despite increases from baseline to 18 percent in the experimental group and to 43 percent in the control group. The corresponding relative risk of hospitalization during that time $(\mathrm{RR}=.41, \mathrm{CI}=.28$ to $.61, \mathrm{p}<.001)$ is equivalent to a 59 percent reduction in an adolescent's likelihood of hospitalization over the six months after an emergency assessment for suicidality.

No statistically significant differences were noted in clinical status (as measured by the CGAS and level of suicidality) identified from the time of entry into the study and over the follow-up period (Table 4). Nor were significant differences noted between groups in the number of return visits to the emergency department during the six-month follow-up period (15 patients in the experimental group and 12 in the control group) or in the mean overall duration of hospitalization per patient hospitalized at the three time points (Table 4). Among the adolescents who were originally hospitalized, only one of the 16 in the experimental group (6 percent) was rehospitalized ( $\mathrm{CI}=-.056$ to .18$)$, compared with five (9.4 percent) of the 53 patients in the control group $(\mathrm{CI}=.016$ to .17$)$. By contrast, among the adolescents who were not originally hospitalized, 12 (9 percent) of the 130 patients in the experimental group (CI=.043 to .14) were subsequently hospitalized one to three times during the six-month follow-up period (a total of 15 hospitalizations), compared with only three (4 percent) of the 75 patients in the control group (CI=-.004 to .08) (a total of three hospitalizations). Finally, no signifi- cant differences were found in the numbers of suicide attempts made during the six-month follow-up period, excluding those at the time of recruitment ( 23 in the experimental group and 14 in the control group).

A coroner's report obtained six months after completion of the study indicated that none of the patients in either group had died.

\section{Discussion and conclusions}

At the time of this study, many psychiatrists in Canada were hospitalizing suicidal adolescents because of long waiting lists. These youths, and their families, often feared the stigma of hospitalization and the associated academic and vocational disruptions. A rapid-response outpatient team was created to treat them while the health care system awaited restructuring.

The lower hospitalization rate in the experimental group and the fact that patients were distributed equally between the two groups suggests that most suicidal adolescents who receive treatment within a rapid-response outpatient model can achieve the same decrease in level of suicidality and increase in level of functioning as those whose treatment relies more heavily on hospitalization. Thus a majority of these patients could be treated within a rapid-response outpatient model without a discernible difference in their clinical outcome as reflected in the measures we used in this study. These findings were reinforced by the absence of significant differences between groups in repeat suicide attempts, return visits to the emergency department, and duration of hospitalization (when that was required) during the follow-up period.

This study overcame the shortcomings of previous studies of suicidal adolescents in that it was prospective, showed clear between-group differences in hospitalization rates, and represented the full spectrum of suicidal adolescents (excluding completed suicides and those who were medically or surgically hospitalized), including both boys and girls and those from every socioeconomic level, who came to an emergency department at every time of each day of the year. In addition, standardized measures were used during a person-to-person inter- 
view, and the rapid-response model could be replicated in many other settings-for example, any setting with an emergency department (such as a hospital) and settings without an emergency department (such as a community clinic) where personnel can nonetheless be available to respond to emergencies.

The study was limited by the fact that the sample included only 88 percent of the suicidal adolescents who were seen in the emergency department, for whom a psychiatrist alone determined the need for hospitalization. Because the study was intended to focus on psychiatric decision making, the suicidal patients who were hospitalized for medical and surgical reasons related to the suicide attempt (12 percent) were excluded-for example, patients whose overdose required ongoing cardiac monitoring and thus admission to a medical ward. Had these patients been included, the intergroup differences might have been diluted.

Preassignment of the treatment conditions was chosen as the most feasible approach to the equal distribution of patients to both groups. This approach probably did not result in any systematic bias in terms of patient selection or information obtained, because every hour of every day of both study years was covered equally by psychiatrists in both groups.

In conclusion, patients who had access to a rapid-response outpatient team were hospitalized less often than those who did not. The outcomes associated with the two treatment models were essentially identical, suggesting that most adolescents-of both genders and from a broad sociodemographic and diagnostic spectrumcan be treated within a simple rapidresponse outpatient team model and thus avoid the stresses associated with hospitalization.

\section{Acknowledgments}

This study was supported by grant 6605 4656-011 from the National Health Research and Development Program. The authors thank Patti Dray, M.A., and Aline Drapeau, Ph.D., for coordinating the assessments; Rick Jane, B.A., Hong Yang, M.Sc., Isabelle Morin, M.Sc., and Elise Mok, M.Sc., for their statistical support; Fiona Key, M.D., Klaus Minde, M.D.,
Mournir Samy, M.D., and Claudine Tremblay, M.D., for their inpatient and outpatient care of the patients; and Eric Fombonne, M.D., Mike Kramer, M.D., Klaus Minde, M.D., Joel Paris, M.D., and Josh Slatkoff, M.Sc., for their critical reviews of the manuscript.

\section{References}

1. Safer DJ: Self-reported suicide attempts by adolescents. Annals of Clinical Psychiatry 9:263-269, 1997

2. Hoyert DL, Kochanek KD, Murphy SL: Deaths: final data for 1997. National Vital Statistics Reports 47:1-104, 1999

3. Statistics Canada: Suicides, and suicide rate, by sex, by age group, in Canadian Statistics. Available at http://www.statcan.ca /english/pgdb/people/health/health01.htm

4. Welch SS: A review of the literature on the epidemiology of parasuicide in the general population. Psychiatric Services 52:368375,2001

5. Gutstein SE, Rudd MD: An outpatient treatment alternative for suicidal youth. Journal of Adolescence 13:265-277, 1990

6. Leslie DL, Rosenheck R: Shifting to outpatient care? Mental health care use and cost under private insurance. American Journal of Psychiatry 156:1250-1257, 1999

7. Greenhill LL, Waslick B: Management of suicidal behavior in children and adolescents. Psychiatric Clinics of North America 20:641-666, 1997

8. Aoun SL, Gregory RJ: Mental disorder of Eskimos seen at a community mental health center in western Alaska. Psychiatric Services 49:1485-1487, 1998

9. Brent DA, Baugher M, Bridge J, et al: Ageand sex-related risk factors for adolescent suicide. Journal of the American Academy of Child and Adolescent Psychiatry 38: 1497-1505, 1999

10. Hughes DH: Can the clinician predict suicide? Psychiatric Services 46:449-451, 1995

11. Patel SP, Gaw AC: Suicide among immigrants from the Indian subcontinent: a review. Psychiatric Services 47:517-521, 1996

12. Shuchman M, Silbernagel KH, Chesney MA, et al: Interventions among adolescents who were violently injured and those who attempted suicide. Psychiatric Services $47: 755-757,1996$

13. Weiner DA, Abraham ME, Lyons J: Clinical characteristics of youths with substance use problems and implications for residential treatment. Psychiatric Services 52: 793-799, 2001

14. Trautman PD, Stewart N, Morishima A: Are adolescent suicide attempters noncompliant with outpatient care? Journal of the American Academy of Child and Adolescent Psychiatry 32:89-94, 1993

15. Gunnell DJ, Brooks J, Peters TJ: Epidemiology and patterns of hospital use after parasuicide in the south west of England.
Journal of Epidemiology and Community Health 50:24-29, 1996

16. Harrington R, Kerfoot M, Dyer E, et al: Randomized trial of a home-based family intervention for children who have deliberately poisoned themselves. Journal of the American Academy of Child and Adolescent Psychiatry 37:512-518, 1998

17. McLeavey BC, Daly RJ, Ludgate JW, et al: Interpersonal problem-solving skills training in the treatment of self-poisoning patients. Suicide and Life-Threatening Behavior 24:382-394, 1994

18. Rotheram-Borus MJ, Piacentini J, Van Rossem R, et al: Enhancing treatment adherence with a specialized emergency room program for adolescent suicide attempters. Journal of the American Academy of Child and Adolescent Psychiatry 35:654-663, 1996

19. Rudd MD, Rajab MH, Orman DT, et al: Effectiveness of an outpatient intervention targeting suicidal young adults: preliminary results. Journal of Consulting and Clinical Psychology 64:179-190, 1996

20. Segal HG, King CA, Naylor MW: Psychosocial functioning of severely disturbed adolescents after short-term hospitalization. Psychiatric Services 46:287-289, 1995

21. Henggeler SW, Rowland MD, Randall J, et al: Home-based multisystemic therapy as an alternative to the hospitalization of youths in psychiatric crisis: clinical outcomes. Journal of the American Academy of Child and Adolescent Psychiatry 38:1331-1339, 1999

22. Costello AJ, Edelbrock CS, Dulcan MK, et al: Report on the NIMH Diagnostic Interview Schedule for Children (DISC). Rockville, Md, National Institute of Mental Health, 1984

23. Diagnostic and Statistical Manual of Mental Disorders, 3rd ed, rev. Washington, DC, American Psychiatric Association, 1987

24. Shaffer D, Gould MS, Brasic J, et al: A Children's Global Assessment Scale (CGAS). Archives of General Psychiatry 40:1228-1231, 1983

25. Chambers WJ, Puig-Antich J, Hirsch M, et al: The assessment of affective disorders in children and adolescents by semistructured interview: test-retest reliability of the Schedule for Affective Disorders and Schizophrenia for School-Age Children, present episode version. Archives of General Psychiatry 42:696-702, 1985

26. Pfeffer CR: The Suicidal Child. New York, Guilford, 1986

27. Hudson WW: The Clinical Measurement Package: A Field Manual. Homewood, Ill, Dorsey, 1982

28. Coddington RD: The significance of life events as etiologic factors in the diseases of children: II. a study of a normal population. Journal of Psychosomatic Research 16: 205-213, 1972 